\title{
Genotypic x Environment Interaction and Stability Analysis in Turmeric (Curcuma longa L.)
}

\author{
Abhishek Pratap Singh ${ }^{1}$, M. D. Ojha ${ }^{2 *}$, V. P. Pandey ${ }^{3}$ and Vijay Kumar ${ }^{2}$ \\ ${ }^{1}$ Horticulture, Krishi Vigyan Kendra, Purnea (Bihar Agricultural University, \\ Sabour, Bhagalpur), India \\ ${ }^{2}$ Nalanda College of Horticulture, Noorsarai (Bihar Agricultural University, \\ Savour, Bhagalpur), India \\ ${ }^{3}$ Narendra Deva University of Agriculture \& Technology, Kumarganj Faizabad-224229, India \\ *Corresponding author
}

\section{A B S T R A C T}

\section{Ke y w o r ds \\ Crop improvement, Curcuma longa, Genotypic $\mathrm{x}$ environment interaction, Stability analysis, Yield}

\section{Article Info}

Accepted:

22 March 2020

Available Online:

10 April 2020

Seventeen genotypes of turmeric (Curcuma longa L.) were grown during two consecutive seasons during 2007-08 and 2008-09 at two locations i.e. Main Experiments Station, Department of Vegetable Science, Narendra Deva University of Agriculture and Technology, Kumarganj, Faizabad, U.P and Krishi Vigyan Kendra, Masodha, Faizabad, U.P, for their stability analysis for yield and yield components like weight of mother rhizome $(\mathrm{g})$, weight of primary rhizomes per plant $(\mathrm{g})$, weight of fresh rhizome per plant (g), rhizomes yield (q/ha), dry matter $(\%)$, curcumin $(\%)$ and oleoresin $(\%)$. Mean square due to environment and linear were expressed highly significant for all the traits except curcumin and oleoresin per cent. Linear components of genotypic $\times$ environment interaction assumed importance for weight of mother rhizome, weight of primary rhizome per plant and dry matter. Genotypic $\times$ Environment interactions were found to be significant for all the characters except rhizome yield, curcumin and oleoresin. The pooled deviation was found to be significant for weight of mother rhizome and weight of fresh rhizome per plant. The genotypes NDH-88 was most desirable for wide range of environment for weight of primary rhizome per plant whereas, NDH-118, NDH- 98 and NDH-79 were suited for adverse environmental conditions and NDH-88, NDH-45, NDH-9 and NDH-74 produced highest yield under favourable environmental conditions. Prabha was most desirable variety for wide range environment for curcumin per cent whereas, NDH-7 and Rajendra Sonia were suited for poor environmental conditions and NDH-18, NDH-14 and NDH-98 produced highest curcumin under favourable environment. The two characters namely weight of primary rhizome and curcumin per cent were characterized as stable traits over the four environments.

\section{Introduction}

Turmeric (Curcuma longa L.) the golden spice of life is one of the most essential spices used as important gradient in culinary and medicinal crop all over the world. The rhizome contains yellow colouring component curcumin (3-9\%), essential oil (5- 
9\%) and oleoresin (3-13\%). Curcumin is gaining more importance in food industries, pharmaceuticals, preservatives and cosmetics. The ban on artificial colour has prompted the use of curcumin as a food colourant. In pharmaceuticals it is valued for the anticancerous, anti-inflammatory, antiseptic, antimicrobial and anti-proliferative activities (Srimal, 1997)(10). It is a tropical crop and needs a warm and humid climate with an optimum temperature of 20 to $30^{\circ} \mathrm{C}$ for normal growth and satisfactory production. It thrives best on sandy, loamy or alluvial, loose, friable and fertile soil rich in organic matter status and having a $\mathrm{pH}$ range of 5.0 to 7.5. The crop cannot withstand water logging. The occurrence of genotype-environment interaction has provided a major challenge for obtaining full understanding of the genetic control of variability. The study of genotypeenvironment interaction in its biometrical aspects is thus important not only from the genetical and evolutionary pain of view, but it is also very relevant to the production problems of agriculture in general plant breeding in particular (Breese, 1969) (1). A variety having wide or good adoptability is one which consistently given superior production over a wide range of environments (Frey, 1964) (5). This combination of stability and performance is very important. Stability is a common practice in trials involving varieties and breeding lines to grow a series of genotypes in a range of different environments. Therefore, the present investigation was conceived with the objective to study thegenotype $\mathrm{x}$ environment interaction and to identify the most productive and stable genotype and environment.

\section{Materials and Methods}

The present experiment was carried out for two consecutive seasons during 2007-08 and 2008-09 at two different locations of main Experiments Station, Department of Vegetable Science, Narendra Deva university of Agriculture and Technology, Kumarganj, Faizabad, U.P and KVK, Masodha, Faizabad, U.P. The experiment comprised of seventeen genotypes (NDH-7, NDH-108, NDH-88, NDH-86, Prabha, NDH-45, NDH-18, NDH-8, NDH-68, NDH-53, NDH-9, NDH-14, NDH74, NDH-79, NDH-98, NDH-118 and Rajendra Sonia) and laid out in complete randomized block design with three replications. The soils of main Experiments Station, Department of Vegetable Science, Narendra Deva university of Agriculture and Technology, Kumarganj, Faizabad, U.P. was silty loam in texture, slightly sodic in reaction with $\mathrm{pH} 8.6$ and low in organic carbon $(0.36 \%)$, available nitrogen $(118.7 \mathrm{~kg} / \mathrm{ha})$ and medium in available $\mathrm{P}(14.9 \mathrm{~kg} / \mathrm{ha})$ and $\mathrm{K}$ $(224.2 \mathrm{~kg} / \mathrm{ha})$ and soils of $\mathrm{KVK}$, Masodha, Faizabad, U.P. farm was sandy loam in texture, slightly saline in reaction with $\mathrm{pH} 7.4$ and medium in organic carbon $(0.50 \%)$, low in available nitrogen (165.4 $\mathrm{kg} / \mathrm{ha})$ and medium in available $\mathrm{P}(16.4 \mathrm{~kg} / \mathrm{ha})$ and $\mathrm{K}$ $(245.2 \mathrm{~kg} / \mathrm{ha})$. Rhizomes of each genotype were planted in the month of June at a spacing of $30 \times 20 \mathrm{~cm}$ and harvested in the month of February. The crop was grown with recommended package of practices. Observation was recorded from five randomly selected plants for weight of mother rhizome (g), weight of primary rhizomes per plant $(\mathrm{g})$, weight of fresh rhizome per plant $(\mathrm{g})$, rhizomes yield (q/ha), dry matter (\%), curcumin (\%) and oleoresin content (\%).The collected pooled were subjected for statistical analysis as per method of Eberhart and Russel (1966) (2).

\section{Results and Discussion}

A highly significant difference was observed among the genotypes for all characters under observation. The differences amongst the environment were also significant for all characters except rhizome yield, curcumin and oleoresin. Genotype $\mathrm{x}$ environment interaction were highly significant for weight 
of mother rhizome, weight of primary rhizomes per plant, weight of fresh rhizomes per plant and dry matter while significant at low probability for plant girth. Environment (Genotype $\mathrm{x}$ Environment) interaction were highly significant for weight of mother rhizome, weight of primary rhizomes per plant, weight of fresh rhizomes per plant and dry matter and non-significant for rhizomes yield, curcumin (\%) and oleoresin (\%) content. The linear component of environment was highly significant for all the characters except rhizome yield, curcumin and oleoresin. The linear component of genotypes $\mathrm{x}$ environment interaction was highly significant for all the characters except rhizome yield, curcumin and oleoresin. The pooled deviations were highly significant for weight of mother rhizome, and weight of fresh rhizomes per plant remaining characters showed non-significant response. Three stability parameters viz., mean (Xi), linear sensitivity coefficient (bi) and non linear sensitivity coefficient $\left(S^{2}\right.$ di) were carried out only for rhizome yield and important yield contributing traits along with quality traits. These characters were weight of mother rhizome, weight of primary rhizomes per plant, weight of fresh rhizome per plant, rhizome yield, oleoresin and dry matter per cent (Table 2). The mean performance over environments showed that weight of mother rhizome varied from 22.39 (R. Sonia) to $192.64 \mathrm{~g}$ (NDH-98). Six genotypes namely, NDH-98, NDH-7, NDH-9, NDH-68, NDH118 and NDH-45 had significantly higher mean for weight of mother rhizome as compared to the general mean. However, genotypes NDH-108, NDH-88, NDH-86, Prabha, NDH-18, NDH-8, NDH-53, NDH-14, NDH-74, R. Sonia and NDH-79 were found significantly inferior from general mean (Table 1). Out of seventeen genotypes, NDH98 and NDH-45 exhibited more than one bi value. Fifteen genotypes had lower linear response. Non-linear sensitivity coefficient
( $\mathrm{S}^{2}$ di) was significant for NDH-7, NDH-108, NDH-86, NDH-45, NDH-18, NDH-8, NDH68, NDH-9 and NDH-98. The rest of the genotypes were characterized by $\mathrm{S}^{2} \mathrm{di}=0$. Two genotypes viz., NDH-98 and NDH-45 had high mean, $(\overline{\mathrm{X}} \mathrm{i})$, bi $>1$ and $\mathrm{S}^{2} \mathrm{di}=0$ which indicated that these genotypes were highly responsive to favourable environments. The genotypes NDH-7, NDH-8, NDH-64 and NDH-118 had high mean value (Xi), bi $=<1$ with $\mathrm{S}^{2} \mathrm{di}=0$ which indicated that this genotype was stable for unfavourable environments. The mean performance of the genotypes over environments (Xi), linear coefficient (bi) and deviation from linearity $\left(\mathrm{S}^{2} \mathrm{di}\right)$ for this character are presented in Table 1. Weight of primary rhizomes per plant ranged from 45.27 (NDH-68) to $157.72 \mathrm{~g}$ (NDH-98). Seven genotypes viz., NDH-88, NDH-45, NDH-79, NDH-98, NDH-9, NDH74 and NDH-118 had significantly higher mean for weight of primary rhizomes per plant than general mean. Ten genotypes namely, NDH-7, NDH-108, R. Sonia, NDH86, Prabha, NDH-18, NDH-8, NDH-68, NDH-53 and NDH-14 had lower mean values for this trait. Out of seventeen genotypes, seven genotypes namely NDH-88, NDH-45, NDH-9, NDH-14, NDH-18, NDH-74 and R. Sonia showed greater than one regression value (bi >1). The non linear deviation from regression coefficient was found significantly different from zero in case of seven entries namely, NDH-86, NDH-45, NDH-68, NDH9, NDH-14, NDH-79 and R. Sonia. Three genotypes NDH-98, NDH-79 and NDH-118 had high mean performance $(\mathrm{Xi}), \mathrm{bi}=<1 \mathrm{~S}^{2} \mathrm{di}$ $=0$ which indicated that these genotypes were stable for unfavourable environments and three genotypes viz., NDH-9, NDH-74 and NDH-45 had high mean performance, bi $>1$ with $S^{2}$ di=0 which indicated that these genotypes were suitable for only favourable environments. NDH-88 showed high mean performance, bi=1 with $\mathrm{S}^{2} \mathrm{di}=0$ which indicated that these genotype were stable 
wide range of environment. On the basis of mean performance over environments, the weight of fresh rhizome per plant varied from 176.76 (NDH-86) to $937.94 \mathrm{~g}$ (NDH-98). Out of seventeen genotypes three genotypes i.e., R. Sonia, NDH-118 and NDH-98 had significantly higher mean values than general mean while, rest of the genotypes had lower mean values than general mean for weight of fresh rhizomes per plant (Table 2). The regression coefficient (bi) was greater than one for eight genotypes while three genotypes showed less than one bi value. The deviation from regression $\left(\mathrm{S}^{2} \mathrm{di}\right)$ was significantly greater than zero in fourteen genotypes viz., NDH-108, NDH-88, NDH-86, NDH-45, NDH-18, NDH-68, NDH-53, NDH-9, NDH14, NDH-74, NDH-79, NDH-98, NDH-118 and R. Sonia while rest of the genotypes had non-significant $\mathrm{S}^{2} \mathrm{di}=0$. Two genotypes namely, NDH-98 and R. Sonia had high mean value bi $>1$ with $S^{2}$ di $=0$ which indicated that this genotypes are suitable for favourable environments. One genotype namely $\mathrm{NDH}-$ 118 showed high mean performance, bi <1 which $S^{2}$ di $=0$ which indicated that these genotypes were suitable for unfavourable environment. The mean performance over all the environments showed that rhizome yield ranged from 232.59 (NDH-86) to $421.79 \mathrm{q} / \mathrm{ha}$ (NDH-98). Nine genotypes namely, NDH118, NDH-98, NDH-79, NDH-74, NDH-14, NDH-9, NDH-68, NDH-8 and NDH-18 had significantly higher mean for rhizome yield as compared to the general mean (Table 2). Out of seventeen genotypes, four genotypes had more than one regression coefficient, while eight genotypes had less than one regression coefficient. Non-linear sensitivity coefficient $\left(\mathrm{S}^{2} \mathrm{di}\right)$ was significant for NDH-14 whereas, rest of the genotypes were characterized by $\mathrm{S}^{2} \mathrm{di}=0$. The genotype NDH-79, NDH-98 and NDH-18 had high mean (Xi), bi $>1$ and $\mathrm{S}^{2} \mathrm{di}=0$ which indicated that genotypes were more responsive for favourable environments. Six genotypes viz., NDH-118, NDH-74,
NDH-14, NDH-9, NDH-68 and NDH-8 had high mean values, bi $<1$ with $S^{2}$ di $=0$ which indicated that these genotypes were suitable for unfavourable environment. The mean performance across the environments showed that dry matter per cent varied from 17.97 (NDH-8) to $28.23 \%$ (NDH-68). Out of seventeen genotypes, six genotypes namely, NDH-88, Prabha, NDH-68, NDH-53, NDH79 and NDH-98 had high mean values for this trait as compared to the general mean (Table 3 ). The regression coefficient (bi) was higher than unity for three genotypes while nine genotypes showed less than one values for bi. The deviation from regression was significant in three genotypes viz., NDH-108, Prabha and NDH-68 while, rest of the genotypes had nonsignificant $\mathrm{S}^{2}$ di. The genotype viz., Prabha, NDH-68 and NDH-53 had high mean (Xi), bi $>1$ with $\mathrm{S}^{2} \mathrm{di}=0$ which indicated that these genotypes were suitable for favourable environment. The genotypes viz., NDH-88, NDH-79 and NDH-98 had high mean value, bi $<1$ and $S^{2}$ di $=0$ were most responsive in unfavourable environments. The curcumin per cent ranged from $3.16 \%$ (NDH-68) to 8.43 (NDH-98) with a general mean of $5.49 \%$. Out of seventeen genotypes, six genotypes showed significantly higher while six genotypes showed significantly lower mean performance values for curcumin per cent (Table 3). The regression coefficient (bi) was higher than units for two genotypes while eleven genotypes showed less than one value for bi. The deviation from regression for genotypes viz., NDH-18 and NDH-98 were significant and for rest the genotypes were non-significant. NDH-53 had high mean, bi = 1 and $\mathrm{S}^{2} \mathrm{di}=0$ thus considered stable genotypes for wide range of environments. Four genotypes viz., Prabha, NDH-18, NDH14 and NDH-98 had high mean (Xi), bi $>1$ with $S^{2} \mathrm{di}=0$ which indicated that these genotypes were suitable for favourable environments. 
Table.1 Estimates of stability parameters for weight of mother rhizome $(\mathrm{g})$ and weight of primary rhizomes/plant (g)

\begin{tabular}{|c|c|c|c|c|c|c|c|}
\hline \multirow[t]{2}{*}{ S.No. } & \multirow[t]{2}{*}{ Genotypes } & \multicolumn{3}{|c|}{ Weight of mother rhizome (g) } & \multicolumn{3}{|c|}{ Weight of primary rhizomes/plant (g) } \\
\hline & & $\overline{\mathbf{X i}}$ & bi & $S^{2} \mathbf{d i}$ & $\overline{\mathbf{X i}}$ & bi & $S^{2}$ di \\
\hline 1. & NDH-7 & 62.52 & -0.15 & $97.76^{* *}$ & 291.72 & 2.18 & $1550.87 * *$ \\
\hline 2. & NDH-108 & 24.44 & 0.23 & $8.59 *$ & 341.57 & 1.57 & 83.49 \\
\hline 3. & NDH-88 & 41.66 & 0.02 & -1.04 & 259.30 & 0.52 & $987.57 *$ \\
\hline 4. & NDH-86 & 23.74 & 0.04 & $53.05 * *$ & 430.73 & 1.06 & -78.06 \\
\hline 5. & Prabha & 24.37 & -0.02 & -3.22 & 279.16 & 2.12 & 170.15 \\
\hline 6. & NDH-45 & 58.40 & 2.4 & $1151.96 * *$ & 289.00 & 1.55 & $940.12 *$ \\
\hline 7. & NDH-18 & 24.45 & -0.06 & $32.82 * *$ & 302.68 & 1.07 & 59.49 \\
\hline 8. & NDH-8 & 52.03 & 0.21 & $145.26 * *$ & 268.32 & -0.92 & $1407.15 * *$ \\
\hline 9. & NDH-68 & 55.29 & -0.26 & $604.96 * *$ & 477.04 & -1.27 & 41.53 \\
\hline 10. & NDH-53 & 29.47 & 0.44 & 4.48 & 241.41 & 1.15 & -89.84 \\
\hline 11. & NDH-9 & 62.64 & -0.19 & $12.02 * *$ & 294.50 & 0.27 & $1511.07 * *$ \\
\hline 12. & NDH-14 & 34.64 & -0.17 & -0.95 & 457.53 & 0.09 & -104.88 \\
\hline 13. & NDH-74 & 22.63 & -0.13 & -1.09 & 281.21 & -1.37 & 1444.92 \\
\hline 14. & NDH-79 & 34.63 & -0.07 & 0.41 & 474.98 & 0.28 & -124.36 \\
\hline 15. & NDH-98 & 192.64 & $15.36 * *$ & $693.92 * *$ & 301.11 & -1.21 & $933.63 *$ \\
\hline 16. & NDH-118 & 52.32 & -0.05 & -71.16 & 479.67 & 1.02 & -147.60 \\
\hline 17. & Rajendra Sonia & 22.39 & -0.16 & -290.36 & 315.12 & 1.87 & $1026.83 *$ \\
\hline & G. mean & 48.13 & 46.72 & - & 304.30 & 1.00 & - \\
\hline & $\mathrm{SE} \pm$ & 0.90 & & - & 6.11 & 0.85 & - \\
\hline
\end{tabular}

*,** Significantly at $5 \%$ and $1 \%$ level, respectively 
Table.2 Estimates of stability parameters for weight of fresh rhizome/plant (g) and rhizome yield (q/ha)

\begin{tabular}{|c|c|c|c|c|c|c|c|}
\hline \multirow[t]{2}{*}{ S.No. } & \multirow{2}{*}{ Genotypes } & \multicolumn{3}{|c|}{ Weight of fresh rhizome/plant (g) } & \multicolumn{3}{|c|}{ Rhizome yield (q/ha) } \\
\hline & & $\overline{\mathbf{X i}}$ & bi & $S^{2}$ di & $\overline{\mathbf{X i}}$ & bi & $\mathbf{S}^{2} \mathbf{d i}$ \\
\hline 1. & NDH-7 & 260.18 & -0.79 & 14.78 & 313.11 & 0.72 & -53.34 \\
\hline 2. & NDH-108 & 216.04 & 0.73 & $1788.07 * *$ & 261.12 & 0.12 & -53.06 \\
\hline 3. & NDH-88 & 255.23 & 0.10 & $2384.58 * *$ & 292.66 & 2.44 & -26.50 \\
\hline 4. & NDH-86 & 176.76 & -0.71 & $9105.34 *$ & 232.59 & -0.50 & -53.02 \\
\hline 5. & Prabha & 228.00 & -0.69 & -234.42 & 318.40 & 0.42 & -54.72 \\
\hline 6. & NDH-45 & 236.52 & 2.43 & $1643.32 * *$ & 313.65 & -0.00 & -53.80 \\
\hline 7. & NDH-18 & 188.40 & -0.14 & $4239.04 * *$ & 349.25 & $2.58^{*}$ & -50.85 \\
\hline 8. & NDH-8 & 249.41 & 0.04 & -265.30 & 335.85 & 0.67 & -30.22 \\
\hline 9. & NDH-68 & 182.89 & 5.10 & $9064.93 * *$ & 325.01 & 0.28 & -21.89 \\
\hline 10. & NDH-53 & 207.65 & 2.08 & $1516.84 * *$ & 267.34 & 0.23 & -46.24 \\
\hline 11. & NDH-9 & 215.70 & 1.61 & $1671.87 * *$ & 363.84 & -0.21 & -47.24 \\
\hline 12. & NDH-14 & 188.94 & 1.08 & $3397.89 * *$ & 362.46 & 0.18 & $125.30 *$ \\
\hline 13. & NDH-74 & 234.38 & 2.00 & $-957.92 * *$ & 324.27 & 0.16 & 24.13 \\
\hline 14. & NDH-79 & 182.49 & -0.36 & $386.43^{* *}$ & 326.41 & $4.51 * *$ & 17.77 \\
\hline 15. & NDH-98 & 936.94 & 3.54 & $18855.45 * *$ & 321.79 & $5.61 * *$ & 11.60 \\
\hline 16. & NDH-118 & 301.66 & -0.38 & $-1212.74 * *$ & 324.91 & -0.01 & -7.09 \\
\hline \multirow[t]{3}{*}{17.} & Rajendra Sonia & 319.30 & 1.33 & $3342.38 * *$ & 314.26 & -0.50 & -39.41 \\
\hline & G. mean & 269.50 & & - & 320.41 & -3.33 & - \\
\hline & $\mathrm{SE} \pm$ & & & - & & & - \\
\hline
\end{tabular}


Table.3 Estimates of stability parameters for dry matter (\%) curcumin (\%) and oleoresin (\%)

\begin{tabular}{|c|c|c|c|c|c|c|c|c|c|c|}
\hline \multirow[t]{2}{*}{ S.No. } & \multirow[t]{2}{*}{ Genotypes } & \multicolumn{3}{|c|}{ Dry Matter } & \multicolumn{3}{|c|}{ Curcumin (\%) } & \multicolumn{3}{|c|}{ Oleoresin $(\%)$} \\
\hline & & $\overline{\mathbf{X i}}$ & bi & $S^{2} d i$ & $\overline{\mathbf{X i}}$ & bi & $\mathbf{S}^{2} \mathrm{di}$ & $\overline{\mathbf{X i}}$ & bi & $S^{2} d i$ \\
\hline 1. & NDH-7 & 18.76 & 1.13 & -0.02 & 7.18 & 0.86 & -0.04 & 1.50 & -0.00 & -0.36 \\
\hline 2. & NDH-108 & 19.15 & 0.25 & $0.58 * *$ & 3.96 & 0.62 & -0.05 & 1.05 & 0.82 & -0.35 \\
\hline 3. & NDH-88 & 24.10 & 0.13 & -0.04 & 4.18 & 0.45 & -0.04 & 5.82 & 0.06 & -0.36 \\
\hline 4. & NDH-86 & 18.98 & 1.39 & -0.06 & 4.24 & 0.94 & -0.05 & 12.67 & 7.84 & 0.71 \\
\hline 5. & Prabha & 24.38 & $5.22 * *$ & $0.30 *$ & 7.56 & 1.10 & -0.05 & 10.84 & -0.34 & -0.36 \\
\hline 6. & NDH-45 & 20.80 & 1.66 & -0.02 & 3.62 & 0.91 & -0.05 & 5.70 & 0.06 & -0.36 \\
\hline 7. & NDH-18 & 18.20 & -0.20 & -0.10 & 7.92 & $2.14 * *$ & -0.04 & 13.35 & -0.38 & -0.36 \\
\hline 8. & NDH-8 & 17.97 & 0.89 & -0.05 & 4.51 & 0.58 & -0.05 & 5.99 & $7.64 * *$ & $1.30 *$ \\
\hline 9. & NDH-68 & 28.23 & $6.26 * *$ & $1.15^{*}$ & 3.16 & 0.73 & -0.00 & 6.56 & 0.81 & -0.34 \\
\hline 10. & NDH-53 & 25.43 & $2.56^{* *}$ & -0.11 & 5.12 & 1.07 & -0.05 & 8.20 & -0.15 & -0.36 \\
\hline 11. & NDH-9 & 20.91 & -0.31 & -0.09 & 5.41 & 0.93 & -0.00 & 10.18 & 0.04 & -0.36 \\
\hline 12. & NDH-14 & 18.74 & -0.61 & -0.06 & 7.12 & 1.80 & -0.04 & 13.59 & 0.03 & -0.36 \\
\hline 13. & NDH-74 & 18.82 & -2.58 & -0.07 & 4.73 & 1.51 & -0.04 & 9.88 & 0.00 & -2.16 \\
\hline 14. & NDH-79 & 21.19 & 0.31 & -0.82 & 5.19 & 0.47 & -0.04 & 9.64 & 0.03 & $0.00 * *$ \\
\hline 15. & NDH-98 & 23.37 & -0.45 & -0.15 & 8.43 & $1.87 *$ & 0.00 & 8.15 & 0.52 & 0.00 \\
\hline 16. & NDH-118 & 18.18 & 0.15 & 0.17 & 3.97 & 0.15 & 0.00 & 10.35 & 0.01 & -0.00 \\
\hline \multirow[t]{3}{*}{17.} & Rajendra Sonia & 19.07 & 1.8 & -0.10 & 6.99 & 0.80 & -0.00 & 9.16 & -0.06 & -3.33 \\
\hline & G. mean & 20.96 & 1.16 & - & 5.49 & 0.82 & - & 8.63 & 0.82 & - \\
\hline & $\mathrm{SE} \pm$ & & & - & & & - & & & - \\
\hline
\end{tabular}

*, ** Significantly at $5 \%$ and $1 \%$ level, respectively 
The genotypes viz., NDH-7 and R. Sonia had high mean value, bi $<1$ and $S^{2}$ di $=0$ were most responsive in unfavourable environments. The oleoresin per cent in various genotypes ranged from 3.05 (NDH$108)$ to $13.59 \%(\mathrm{NDH}-14)$ with a general mean $8.63 \%$. Out of seventeen genotypes, nine genotypes showed significantly higher mean performance while two genotypes showed lower mean values for oleoresin $\%$ (Table 3). The regression coefficient (bi) was higher than unity for two genotypes while six genotypes showed less than one value for bi. The deviation from regression was found nonsignificant except for NDH-8. Non-linear sensitivity coefficient $\left(\mathrm{S}^{2} \mathrm{di}\right)$ was significant for NDH-8 and NDH-79 whereas, rest of the genotypes were characterized by $\mathrm{S}^{2} \mathrm{di}=0$. The genotype NDH-86 had high mean $(\overline{\mathrm{X}} \mathrm{i})$, bi $>1$ and $S^{2}$ di $=0$ which indicated that these genotypes were suitable for favourable environment. The genotypes viz., Prabha, NDH-18, NDH-9, NDH-14 and NDH-118 had high mean $(\mathrm{Xi})$, bi $=<1$ and $\mathrm{S}^{2} \mathrm{di}=0$ which indicated that these genotypes were suitable for unfavourable environments.

\section{Acknowledgement}

The authors are thankful to Dr. J. Dixit, Professor, Department of Vegetable Science, N.D.U.A\& T. Kumargang, Faizabad for providing facilities and Dr. Mithlesh Pandey, Programme Coordinator, KVK Masodha, Faizabad for encouragement.

\section{References}

Breese, E.L. 1969. The measurement and significant of genotype environment interaction in grasses. Heredity 24: 26-44.

Eberhart Eberhart, S.A. and Russel, W.A. 1966. Stability parameters for comparing varieties. Crop Sci. 6: 36-40.

Freeman Freeman, G.H. 1973. Statistical methods for analysis of genotypeenvironment interaction. Heredity 31 : 339-354.

Freeman Freeman, G.H. and Perkins, J.M. 1971. Environmental and genotypeenvironmental components of variability. VIII. Relation between genotypes grown in different environments and measure of these environments. Heredity27: 15-23.

Frey Frey, K.J. 1964. Adaptation reaction in oat strains selected under stress and nonstress environmental conditions. Crop Sci., 4: 55-58.

Kumar Kumar, K.S. and Yadav, D.S. 2001. Adaptability of turmeric genotypes in acid hill soils of Manipur. Ind. J. Hill Farming 14 (2): 147-149.

Kumar Kumar, N.A. and Sankaran, P.G. 1998. Instability in turmeric (Curcuma longa L.) production in India. J. Spices Aromatic Crops 7(1): 19-22.

Singh Singh, D.P., and Tiwari, R.S. 1995. Path analysis in turmeric (Curcuma longa L.), Recent Hort. 2(2): 113-116.

Singh Singh, J.P., Singh, M.K., Singh, P.K. and Singh, R.D. 1995. Phenotypic stability in turmeric (Curcuma longa L.). Ind. Cocoa, Arecanut Spices J. 19(2): 4042.

Srimal Srimal, R.C. 1997. Turmeric: a brief review of medicinal properties. Fitoterapia, 68(6): 483-493.

\section{How to cite this article:}

Abhishek Pratap Singh, M. D. Ojha, V. P. Pandey and Vijay Kumar. 2020. Genotypic x Environment Interaction and Stability Analysis in Turmeric (Curcuma longa L.). Int.J.Curr.Microbiol.App.Sci. 9(04): 2656-2663. doi: https://doi.org/10.20546/ijcmas.2020.904.317 